\title{
The Clinical Correlations between Diabetes, Cigarette Smoking and Obesity on Intervertebral Degenerative Disc Disease of the Lumbar Spine
}

\author{
Ande M. Jakoi ${ }^{1}$, Gurpal Pannu ${ }^{2}$, Anthony D’Oro ${ }^{1}$, Zorica Buser ${ }^{1}$, Martin H. Pham ${ }^{3}$, Neil N. Patel ${ }^{1}$, \\ Patrick C. Hsieh ${ }^{3}$, John C. Liu ${ }^{3}$, Frank L. Acosta ${ }^{3}$, Raymond Hah ${ }^{1}$, Jeffrey C. Wang ${ }^{1}$ \\ ${ }^{I}$ Department of Orthopaedic Surgery, University of Southern California, Los Angeles, CA, USA \\ ${ }^{2}$ Department of Orthopaedic Surgery, Drexel University, Philadelphia, PA, USA \\ ${ }^{3}$ Department of Neurological Surgery, University of Southern California, Los Angeles, CA, USA
}

Study Design: Retrospective analysis of a nationwide private insurance database. Chi-square analysis and linear regression models were utilized for outcome measures.

Purpose: The purpose of this study was to investigate any relationship between lumbar degenerative disc disease, diabetes, obesity and smoking tobacco.

Overview of Literature: Diabetes, obesity, and smoking tobacco are comorbid conditions known to individually have effect on degenerative disc disease. Most studies have only been on a small populous scale. No study has yet to investigate the combination of these conditions within a large patient cohort nor have they reviewed the combination of these conditions on degenerative disc disease.

Methods: A retrospective analysis of insurance billing codes within the nationwide Humana insurance database was performed, using PearlDiver software (PearlDiver, Inc., Fort Wayne, IN, USA), to identify trends among patients diagnosed with lumbar disc degenerative disease with and without the associated comorbidities of obesity, diabetes, and/or smoking tobacco. Patients billed for a comorbidity diagnosis on the same patient record as the lumbar disc degenerative disease diagnosis were compared over time to patients billed for lumbar disc degenerative disease without a comorbidity. There were no sources of funding for this manuscript and no conflicts of interest.

Results: The total number and prevalence of patients (per 10,000) within the database diagnosed with lumbar disc degenerative disease increased by $241.4 \%$ and $130.3 \%$, respectively. The subsets of patients within this population who were concurrently diagnosed with either obesity, diabetes, tobacco use, or a combination thereof, was significantly higher than patients diagnosed with lumbar disc degenerative disease alone $(p<0.05$ for all). The number of patients diagnosed with lumbar disc degenerative disease and smoking rose significantly more than patients diagnosed with lumbar disc degenerative disease and either diabetes or obesity $(p<0.05)$. The number of patients diagnosed with lumbar disc degenerative disease, smoking and obesity rose significantly more than the number of patients diagnosed with lumbar disc degenerative disease and any other comorbidity alone or combination of comorbidities $(p<0.05)$. Conclusions: Diabetes, obesity and cigarette smoking each are significantly associated with an increased diagnosis of lumbar degenerative disc disease. The combination of smoking and obesity had a synergistic effect on increased rates of lumbar degenerative disc disease. Patient education and preventative care is a vital goal in prevention of degenerative disc disease within the general population.

Keywords: Degenerative disc disease; Lumbar spine; Tobacco use; Obesity; Diabetes mellitus

\footnotetext{
Received Oct 8, 2016; Revised Nov 29, 2016; Accepted Dec 13, 2016

Corresponding author: Ande M. Jakoi

Department of Orthopaedic Surgery, University of Southern California,

1450 San Pablo Street, Suite 5400, Los Angeles, CA 90033, USA

Tel: +1-323-865-6838, Fax: +1-323-442-5301, E-mail: ajakoi22@gmail.com
} 


\section{Introduction}

Low back pain is a major social and economic burden on the population, resulting in billions of dollars in payments yearly in the United States alone and affecting 15\% to $45 \%$ of the population annually [1-3]. There is a substantial number of new diagnoses of back pain yearly and $1 \%$ to $2 \%$ of the United States adult population is disabled secondary to back pain yearly [4-6]. The estimated total cost of direct medical expenditures in the United States for spine care and related disabilities from spinal pathology in 2006 was more than $\$ 85$ billion and the data suggest the use and cost of spine care have continued to increase in recent years [4-8]. Although the etiology of low back pain remains multifactorial, degenerative changes in the intervertebral discs of the spine have been strongly associated with the onset of pain [1,9-11].

The World Health Organization defines obesity as a body mass index (BMI) of 30 or above [12]. Obesity is a serious public health problem worldwide and its prevalence is rapidly increasing [7]. Obesity has been shown to increase the risks of cardiovascular disease, diabetes, osteoarthritis, and spine disease [13-15]. Obesity has been implicated as a risk factor of lumbar disc degeneration, but previous epidemiological studies have conflicting associations [16].

Diabetes mellitus (DM) is a chronic condition associated with high blood glucose levels resulting from insulin secretory defects and insulin resistance. Elevated glucose concentrations have been associated with an increased risk of disc degeneration by directly influencing disc cell proliferation and survival [17-19].

More than one-third of the population of industrialized countries smoke and in the United States, over 20\% of the adult population admits to smoking [20]. Highly publicized are the respiratory and cardiovascular effects of tobacco use, but smoking can cause many orthopaedic issues, including delayed fracture-healing, decreased bone density, risk of nonunion of fractures and spinal fusions, as well as delayed or poor wound healing [21-23].

Intervertebral disc degeneration involves an imbalance in anabolic and catabolic activity within the annulus fibrosus and nucleus pulposus cells [24-28]. Many factors influence the extracellular environment around intervertebral disc cells including nutrient levels, mechanical loading, and the chemical environment. Alteration in disc cellular viability and activity influences the ability of an- nulus fibrosus and nucleus pulposus cells to produce extracellular matrix components and maintain tissue health [26].

There have been several studies correlating degenerative disc disease to cigarette smoking, obesity or diabetes on a microscopic level, but no population level study evaluating their effect both independently and synergistically.

\section{Materials and Methods}

Within the PearlDiver Patient Record Database (PearlDiver Technologies Inc., Warsaw, IN, USA; http://www.pearldiverinc.com/), International Classification of Diseases, ninth edition (ICD-9) insurance billing codes were used to identify the annual trends of patients diagnosed with lumbar disc degeneration with and without concurrent diagnoses of obesity, diabetes, or tobacco use. We queried the subset of patients within the database that is covered by Humana (Humana Inc., Louisville, KY, USA; https:// www.humana.com/), a private health insurance company that covers nearly 20 million patients across the United States.

Codes ICD-9-D-722.73 and ICD-9-D-722.52 were used to identify the number of patients diagnosed with lumbar disc degenerative disease, with or without myelopathy. The lumbar disc degenerative disease population was then split into two cohorts: those with concurrent diagnoses of tobacco use, diabetes, and/or obesity, and those without those diagnoses. To identify patients diagnosed as having a tobacco use disorder, ICD-9-D-V15.82, was used. Patients who were diagnosed with obesity and morbid obesity were identified with the codes ICD-9-D-278.00 and ICD-9-D-278.01 and analyzed collectively in a group of patients referred to throughout this study simply as obese. Finally, patients diagnosed with type 1 or type 2 diabetes, controlled or uncontrolled, were identified with codes ICD-9-D-250.00, ICD-9-D-250.01, ICD-9-D-250.02, and ICD-9-D-250.03. These patients were also analyzed collectively, in a group of patients considered throughout this paper simply as diabetic.

To qualify to be included in the group "lumbar disc degenerative disease+obesity," for example, patients must have been diagnosed as obese on the same record as their disc degenerative disease diagnosis. To qualify to be included in the group "lumbar disc degenerative disease, non-obese," patients could never have been diagnosed as obese throughout the entire span of the database. For 
groups in which patients had multiple concurrent diagnoses, such as obesity and diabetes, both of those diagnoses similarly had to occur on the same record as the lumbar disc degenerative disease diagnosis. Patients can never have been diagnosed with either to qualify to be included in the analog "non-obese, non-diabetic" group.

For each of the patient populations described above, we tracked the total number of patients who were diagnosed with each code from the beginning of 2007 until the end of 2014. We compared the trends over time between patients with and without concurrent diagnoses of tobacco use, diabetes, or obesity using chi-squared analysis and linear regression. Significance was set at a $p$-value less than 0.05. Demographic information including gender, region, race, and age was collected and analyzed as well.

This retrospective cohort study was deemed exempt from Institutional Review Board review as all patient information was de-identified.

\section{Results}

From 2007 until 2014, the number of people within the Humana database diagnosed with lumbar disc degenerative disease rose $241.1 \%$, from 82,129 to 280,399 . When adjusted for the growth of population within the database, the prevalence per 10,000 patients increased $130.3 \%$, from 128.63 in 2007 to 296.27 in 2014. The prevalence of males and females diagnosed with lumbar disc degenerative disease was roughly equivalent from 2007 until the first quarter of 2015.

Between patients diagnosed as obese, diabetic, or as smokers, smokers were the least prevalent within the database. However, smokers increased in prevalence more than any of the other populations from 2007 through 2014, having risen $381.7 \%$ from 59.39 to 286.15 . The most populous age group in this population was between 70 and 74 years old.

Patients diagnosed as diabetic were the most prevalent but increased in prevalence the least, having risen $81.1 \%$ from 567.78 to $1,028.01$ patients per 10,000 from 2007 through 2014. The prevalence of obese patients rose $214.1 \%$, from 157.76 to 495.53 . For both of these populations, the most populous age group was 60-64 years old.

The number of patients diagnosed with the different possible combinations of two of the above three conditions also rose measurably between 2007 and 2014. The prevalence of patients diagnosed with both smoking and obesity on the same record were the least prevalent combination but increased the most, from 3.66 in 2007 to 25.67 in $2014-$ a $601.1 \%$ increase. The prevalence of patients diagnosed with both obesity and diabetes on the same record were the most prevalent but increased in prevalence the least, from 46.09 in 2007 to 135.94 in 2014-a 195.0\% increase. The prevalence of patients diagnosed with both smoking and diabetes on the same record rose $377.2 \%$, from 10.67 out of 10,000 patients in 2007 to 50.92 in 2014.

The raw total number of each of these patient groups increased markedly as well, and these results are summarized in Table 1.

Of those patients diagnosed with lumbar disc degenerative disease, two cohorts of patients were analyzed: those with a concurrent diagnosis of smoking, diabetes, and/or obesity, and those without a concurrent diagnosis (or collection of diagnoses). In every case, patient populations with concurrent diagnoses increased in total number and in prevalence significantly more than patient populations without those diagnoses from 2007 through 2014.

Patients diagnosed with obesity at the same time as their disc degenerative disease diagnosis rose significantly more between 2007 and 2014 than did patients who never were diagnosed as obese $(p<0.05)$. The prevalence of patients diagnosed with obesity and lumbar disc degenerative disease rose $517.9 \%$, from 1.22 to 7.54 out of 10,000 patients (Fig. 1). This increase was nearly five times the increase of patients not diagnosed with obesity, whose prevalence per 10,000 patients rose $108.0 \%$, from 100.16 in 2007 to 208.37 in 2014.

Patients diagnosed as having a tobacco use disorder at the same time as their disc degenerative disease diagnosis also rose significantly more between 2007 and 2014 than patients who were never diagnosed as smokers $(p<0.05)$. The prevalence of patients with disc degenerative disease who were also smokers rose $627.2 \%$ from 2007 to 2014 (from 0.40 to 2.88 out of 10,000 ) compared to just $117.6 \%$ (108.12 to 235.25) for the population never diagnosed with smoking (Fig. 2).

Furthermore, the subset of patients diagnosed with lumbar disc degenerative disease and diabetes increased significantly more than patients diagnosed as having lumbar disc degenerative disease but no diabetes $(p<0.05)$. The prevalence of patients with a lumbar disc degenerative disease diagnosis but no diabetes diagnoses rose $126.2 \%$ from 80.32 in 2007 to 181.67 in 2014, while the number of patients with a concurrent diabetes diagnoses 


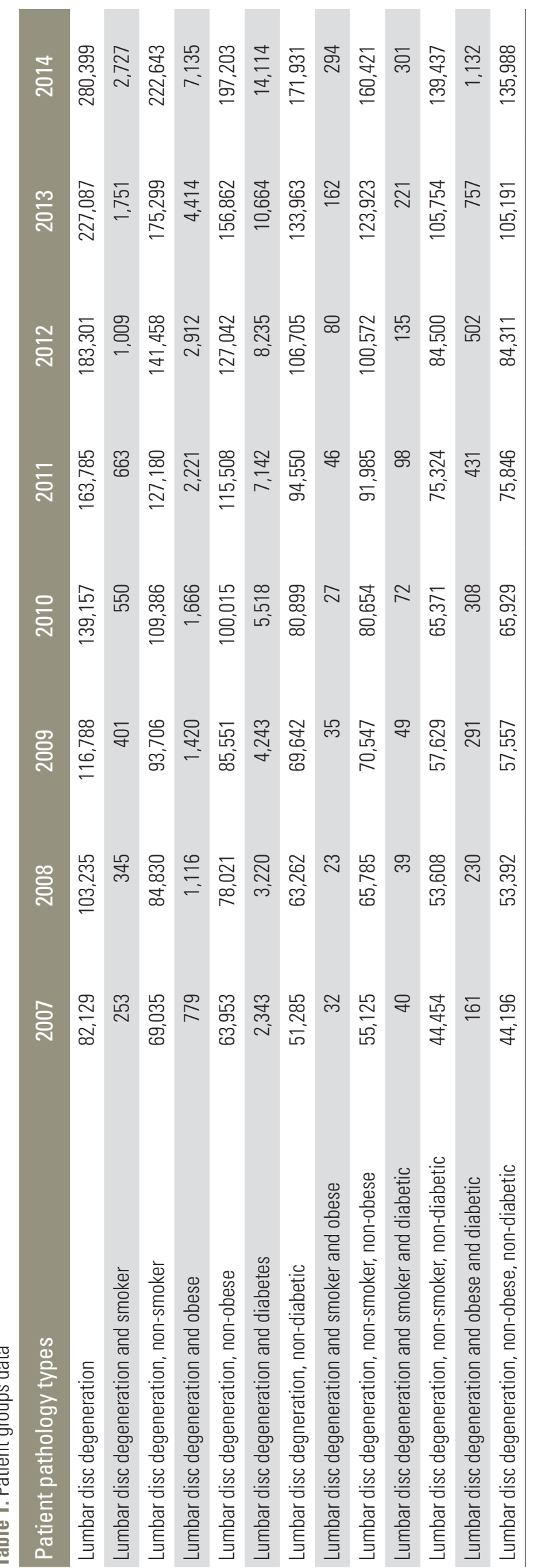

rose $306.4 \%$, from 3.67 to 14.91 out of 10,000 , nearly a three-fold difference (Fig. 3). Gender appeared to make no significant difference on the increase in prevalence among the diabetic and non-diabetic patients diagnosed with lumbar disc degenerative disease.

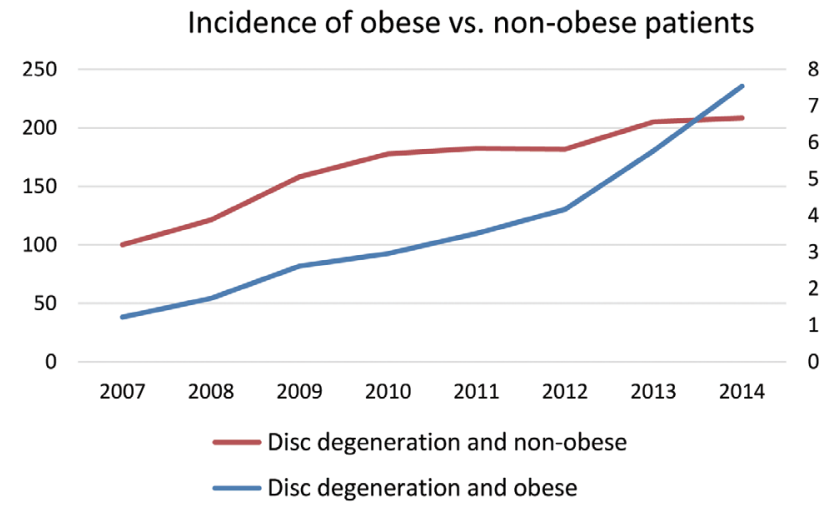

Fig. 1. Incidence of obese vs. non-obese patients with degenerative disc disease per 10,000 patients.

Incidence of smokers vs. non-smokers

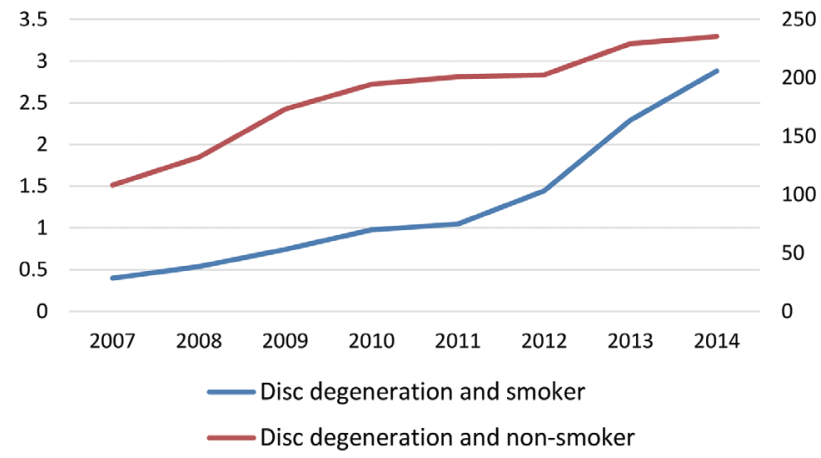

Fig. 2. Incidence of patients who smoke vs. non-smokers with degenerative disc disease per 10,000 patients.

Incidence of diabetic vs. non-diabetic patients

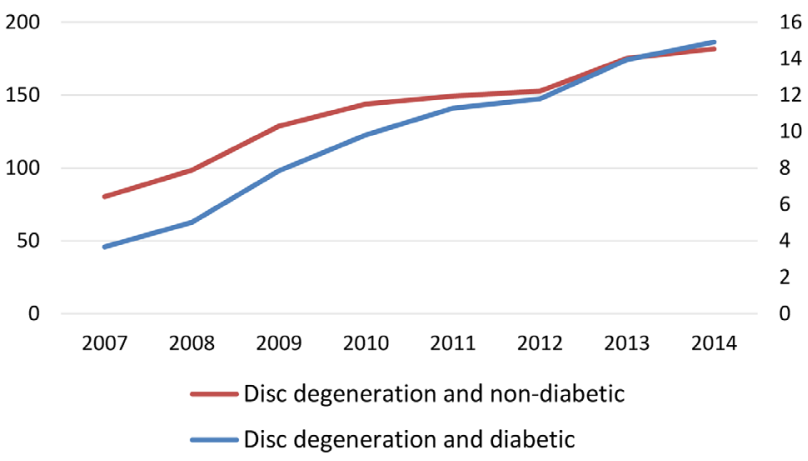

Fig. 3. Incidence of diabetic vs. non-diabetic patient with degenerative disc disease per 10,000 patients. 
Chi-squared analysis revealed that patients diagnosed with lumbar disc degenerative disease and smoking on the same record increased significantly more than the number of patients diagnosed with lumbar disc degenerative disease and either obesity or diabetes $(p<0.05)$. Patients diagnosed with lumbar disc degenerative disease and obesity increased significantly more than the number of patients diagnosed with lumbar disc degenerative disease and diabetes $(p<0.05)$.

The data also demonstrates that lumbar disc degenerative disease patients with a combination of comorbid diagnoses increased significantly more from 2007 through 2014 than patients without either of the comorbid diagnoses. The largest increase in prevalence was seen in the patient group diagnosed with lumbar disc degenerative disease, obesity, and smoking all within the same patient record. The prevalence of this population increased $519.8 \%$ from 0.05 patients per 10,000 in 2007 to 0.31 in 2014 (Fig. 4). The prevalence of patients diagnosed with lumbar disc degenerative disease, diabetes, and smoking rose $407.7 \%$, from 0.06 to 0.32 patients per 10,000 in the database (Fig. 5). Finally, the number of patients diagnosed with lumbar disc degenerative disease, obesity, and diabetes, increased in prevalence by $374.4 \%$, from 0.25 to 1.20 (Fig. 6).

The number of patients diagnosed with lumbar disc degenerative disease, obesity and smoking increased more than patients diagnosed with lumbar disc degenerative disease and diabetes $(p<0.05)$. Interestingly, the number of patients diagnosed with lumbar disc degenerative disease, obesity and diabetes increased at a slower rate than patients diagnosed with lumbar disc degenerative disease and obesity $(p<0.05)$ alone. More, the number of patients diagnosed with lumbar disc degenerative disease, smoking, and diabetes increased less than the number patients diagnosed with lumbar disc degenerative disease and smoking alone $(p<0.05)$. This all suggests that diabetes had the least significant effect on the increased prevalence rate.

There were $8,683,726$ males in the database and $11,225,017$ females in the database. Even after adjusting for the difference between males and females in the database, more females were diagnosed with lumbar degenerative disc disease per every 10,000 males, there were 440.5 patients with lumbar disc degenerative disease and per every 10,000 females, there were 474.4 patients with lumbar disc degenerative disease. Similar trends were true among obese patients with lumbar disc degeneration (females 11.5 to males 8.9 per 10,000). However, the opposite trend was true among smokers and diabetic patients, for whom

Incidence of obese and smoking patients compared to non-obese, non-smoking patients

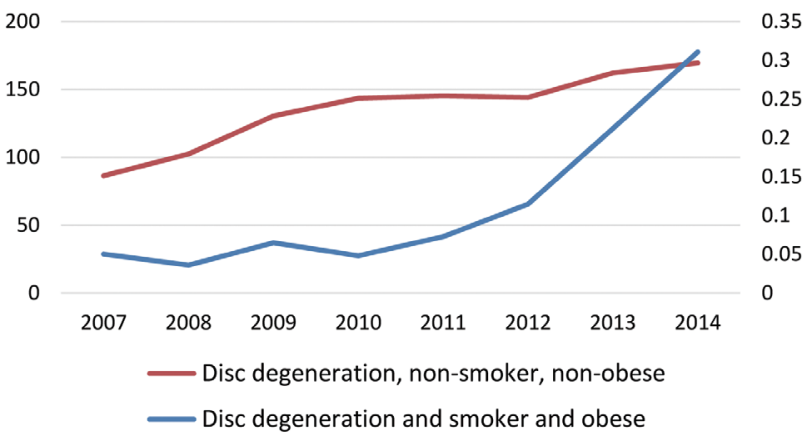

Fig. 4. Incidence of obese patients who smoke vs. non-obese, nonsmoking patients with degenerative disc disease per 10,000 patients.

Incidence of diabetic and smoking patients compared to non-diabetic, non-smoking patients

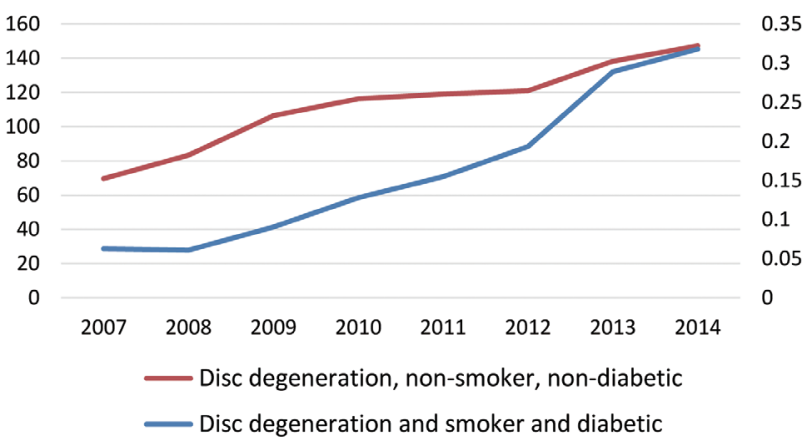

Fig. 5. Incidence of diabetic patients who smoke vs. non-diabetic, non-smoking patients with degenerative disc disease per 10,000 patients.
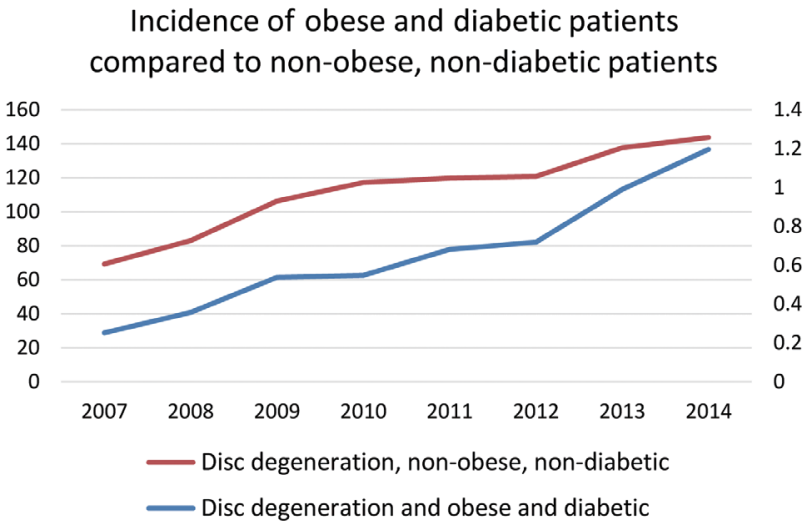

Fig. 6. Incidence of diabetic, obese patients with degenerative disc disease vs. non-diabetic, non-obese patients with degenerative disc disease per 10,000 patients. 
males were more frequent than females. For diabetic patients, the difference was minimal (females $22.7 \%$ to males 24.6 per 10,000), and for smokers, the difference was most pronounced (females 3.4 to males 4.9 per 10,000).

Ages 65-69 were the most populous cohort of patients with lumbar disc degenerative disease $(203,660)$, but after adjusting for the number of patients in each age range within the entire database, ages 60-64 were the most frequent per 10,000 patients in that age range, followed by age 55-59, then 65-69. After adjusting for database demographics, patients aged 60-64 were the most frequently diagnosed with smoking, obesity, or diabetes with lumbar disc degenerative disease.

The database consists of many more patients in the South than any other region in the United States (Northeast, Midwest, or West), but even after adjusting for the number of patients in each region in the database, patients from the South were most frequently diagnosed with lumbar disc degenerative disease $(533.7 / 10,000)$ and patients from the Northwest were diagnosed the least $(136.0 / 10,000)$. Patients from the Northeast were most frequently diagnosed with smoking and disc degenerative disease $(4.44 / 10,000)$, followed closely by the South $(4.38 / 10,000)$. Patients from the South were most frequently diagnosed with smoking or diabetes and lumbar disc degenerative disease.

\section{Discussion}

Given the significant socioeconomic burden of lower back pain on the population and its strong association with intervertebral disc degeneration and disease, there remains considerable interest in identifying factors associated with the development of this degenerative process [1,9-11]. Previous studies have attempted to analyze the link between lower back pain and various comorbid conditions, including smoking [29-31], obesity [32,33], and DM $[17,18]$. These reports range from biomechanical studies to systematic reviews of the literature. Several larger scale studies have attempted to investigate "lower back pain" as opposed to the specific diagnosis of "lumbar disc degeneration." To our knowledge, this is the first report to analyze the relationship between smoking, diabetes and obesity, both independently and synergistically, and the development of lumbar disc degenerative disease at the epidemiologic level. By inclusion of a very large database of almost 20 million patients, we aimed to avoid limita- tions of previous studies, including low study power and the inherent variability in data collection associated with systematic review of literature.

Though the exact pathophysiology of disc degeneration continues to be investigated, it has been demonstrated that the health of the intervertebral disc is maintained by a complex balance of anabolic and catabolic activity within cells of the annulus fibrosis and nucleus pulposus [2428]. There currently exist two primary theories regarding the mechanism with which tobacco use may contribute to intervertebral disc degeneration. The primary proposed mechanism involves malnutrition of the disc caused primarily by tobacco-related anoxia and vascular disease. Malnutrition of the disc has previously been shown to be strongly associated with disc degeneration $[31,34]$ and vascular disruption of this nutritional supply is thought to play a key role [35-38]. Animal studies have demonstrated a reduction in vertebral body blood flow after administration of nicotine [37-39]. The increased blood concentration of carbon monoxide in smokers results in a decreased oxygen-carrying capacity of hemoglobin and this likely represents a further insult to the intervertebral disc's nutritional pathway [40,41].

Alternatively, it has been proposed that chemical compounds found in cigarette smoke may directly affect cell viability and activity in the intervertebral disc $[42,43]$. At the microscopic level, human intervertebral disc cells exposed to tobacco smoke demonstrate significant morphologic changes that appear to be dose-dependent [44]. Beyond these structural changes, exposure to cigarette smoke has been shown to increase cell production of interleukin $1 \beta$, leading to the upregulation of matrixdegrading metalloproteinases $[26,45,46]$. While debate continues regarding the exact mechanism of tobaccorelated disc degeneration, evidence suggests a common end result. Gross examination of intervertebral disc tissue exposed to cigarette smoke demonstrates necrosis and fibrosis involving the nucleus pulposus and alteration in the layered composition of the annulus fibrosis [38,47].

Several reports have demonstrated an association between smoking and disc degeneration using secondary markers, such as radiographic findings or eventual surgical intervention. Utilizing magnetic resonance imaging (MRI), Battie et al. [29] compared the lumbar disc health of twenty pairs of identical twins discordant for tobacco exposure. With respect to disc height and intensity, the authors reported $18 \%$ greater mean disc degeneration 
scores in the smoking group. Of note, these findings were consistent throughout the lumbar spine, indicating a systemic effect and decreasing the likelihood of a physical loading-related confounding variable. After comparing the smoking history of 205 patients that underwent surgical intervention of cervical or lumbar disc disease with an age and sex-matched control population, An et al. [30] reported a significantly greater rate of tobacco use in both the cervical and lumbar disease group. More recently, Mattila et al. [31] reported on a prospective, 11-year follow-up of more than 57,000 adolescents in an attempt to identify risk factors for lumbar discectomy in this young and relatively healthy population. The authors concluded that, with regards to male subjects, daily smoking was the strongest risk factor for lumbar discectomy [31].

However, not all studies have found a significant correlation between smoking and the development of disc degenerative disease. Gore at al. [48] compared lateral cervical radiographs of 50 smokers and 50 non-smokers. The study failed to find a statistically significant difference in degeneration rates, but the authors admit that plain radiography is not the ideal imaging modality to evaluate disc degeneration. After systematic review of 38 epidemiologic studies, Goldberg et al. [49] concluded that while there exists strong support for the association between smoking and non-specific back pain, too few studies have been performed to confidently link smoking with the other studied end points, such as disc degeneration, herniation, and sciatica. These reports emphasize the difficulty in drawing association, let alone causation, in the setting of numerous confounding variables.

Our findings suggest a strong correlation between lumbar disc degenerative disease and tobacco use. The prevalence of lumbar disc degenerative disease in smokers rose $627.2 \%$, representing a nearly 6 -fold increase compared to the non-smoking population.

Our results support a link between lumbar disc degenerative disease and obesity. The prevalence of patients with both lumbar DDD and obesity increased more than 5 times greater than the prevalence of patients with disc degenerative disease without obesity. The prevalence of obesity is high, exceeding $30 \%$ in the United States, and remains a major public health issue [50]. "Damaging loading," perhaps similar to that seen in overweight and obese individuals, has been shown to affect intervertebral cell viability, synthesis and matrix remodeling [51]. Excessive biomechanical loading, as a result of both heavy lifting and obesity, has been linked to disc disease and herniation [52-55]. Obesity has been referred to as some authors as a systemic inflammatory condition, resulting in increased inflammatory markers and the development of atherosclerosis [56,57]. This could conceivably represent a vascular insult to the intervertebral disc. Early reports, based on plain film findings, suggested a possible association between increased BMI and lumbar degeneration [58]. More recently, Samartzis et al. [59] reviewed lumbar MR studies of more than 2,500 individuals and reported a significant association between the presence, extent and severity of disc degenerative changes and increasing body weight. Furthermore, obesity may serve as predictor for hospitalization secondary to disc disorders [60]. Takatalo et al. [61] reported an association between abdominal obesity and disc degeneration in 21-year-old males, suggesting a more rapid effect than previously thought. However, the exact mechanism with which obesity may correlate with disc degeneration is poorly understood and other reports have failed to independently correlate obesity with disc degeneration and disease [33].

Previous studies have suggested that diabetes may negatively affect intervertebral disc health. The accumulation of advanced glycation end products in critical disc proteins, such as aggrecan and collagen, results in abnormal turnover and increasing non-enzymatic cross-linking [62]. Metz et al. [63] revealed a strikingly increased rate of tissue cross-linking in the intervertebral discs of rats with increased glycated hemoglobin (HgbAlc) compared to a healthy control group. Increased cross-linking leads to increased stiffness and brittleness, resulting in an intervertebral disc that is may be more susceptible to mechanical damage $[64,65]$. The well-reported microangiopathy associated with diabetes likely further diminishes the flow of nutrients to the already tenuously supplied intervertebral disc [66]. Sakellaridis [18] reported a significantly higher rate of diabetes in patients undergoing lumbar discectomy compared to patients undergoing elective, non-spine related interventions. Furthermore, diabetic patients have been shown to have significantly decreased disc height, in comparison to their non-diabetic counterparts [67]. These findings suggest important clinical implications of molecular-level changes seen in the diabetic intervertebral disc.

Each co-morbidity was found to have a correlation with an increased likelihood for a diagnosis of degenerative disc disease, but smoking and obesity were found to have 
a synergistic effect on the likelihood of degenerative disc disease. Videman et al. [55] discovered the combination of higher occupational lifting and more smoking during follow-up were predictors of increased disc height reduction in a longitudinal study assessing quantitative MRI measures of disc degeneration in middle-aged 134 monozygotic male twins. Leboeuf-Yde et al. [33] evaluated the association of self-reported physical hard labor, smoking and obesity in four hundred and twelve 40-year old Danes with MRI findings, back pain and a vertebral inflammatory process. They found that there were no significant associations between the single variables and intervertebral disc degeneration. However, they did find that hard physical labor in combination with either heavy smoking or obesity was strongly associated with a vertebral inflammatory process [33].

Limitations of our study include those of any database study. There is a possibility of coding bias due to the somewhat ambiguous nature of Current Procedural Terminology coding. It is also difficult to know if physicians were coding appropriately for lumbar degenerative disc disease at the time of the office visit or if the disease had been present prior to coding for some time and thus correlation with any of the comorbidities may be miscalculated. With regards to the limitations of the database used, we obtained data from a large private insurer (Humana) with customers nationwide, but no other insurers, and the Humana patient population may be distinct from other insurer's populations or the uninsured.

\section{Conclusions}

This study confirms, on a large scale patient population model, that diabetes, obesity and cigarette smoking each are significantly associated with an increased diagnosis of lumbar degenerative disc disease. Previous literature had found that correlation on a smaller population level and only individually among the comorbidities. This study also discovered that the combination of these morbidities either in different pairings or all three had a synergistic effect on increased rates of lumbar degenerative disc disease. Smoking and obesity, above all, had the highest impact and most dramatic synergistic effect leading to increased rates of lumbar degenerative disc disease. Future studies will need to correlate these risk factors further and potentially will involve prospective studies. Nevertheless, this study is a lead in to support the idea of patient educa- tion and preventative care as having a potential impact in prevention of degenerative disc disease within the general population.

\section{Conflict of Interest}

No potential conflict of interest relevant to this article was reported.

\section{References}

1. Kelsey JL, Mundt DF, Golden AL. Epidemiology of low back pain. In: Malcolm JI, editor. The lumbar spine and back pain. Vol. 4. New York: Churchill Livingstone; 1992. p.537-49.

2. Frymoyer JW, Cats-Baril WL. An overview of the incidences and costs of low back pain. Orthop Clin North Am 1991;22:263-71.

3. Katz JN. Lumbar disc disorders and low-back pain: socioeconomic factors and consequences. J Bone Joint Surg Am 2006;88 Suppl 2:21-4.

4. Friedly J, Standaert C, Chan L. Epidemiology of spine care: the back pain dilemma. Phys Med Rehabil Clin N Am 2010;21:659-77.

5. Andersson GB. The epidemiology of spinal disorders. In: Frymoyer JW, editor. The adult spine: principles and practice. 2nd ed. Philadelphia: Lippincott-Raven; 1997. p.107-46.

6. Deyo RA, Mirza SK, Martin BI. Back pain prevalence and visit rates: estimates from U.S. national surveys, 2002. Spine (Phila Pa 1976) 2006;31:2724-7.

7. Katz S. The burden of musculoskeletal diseases in the United States. Rosemont: Bone and Joint Decade, American Academy of Orthopaedic Surgeons; 2008.

8. Luo X, Pietrobon R, Sun SX, Liu GG, Hey L. Estimates and patterns of direct health care expenditures among individuals with back pain in the United States. Spine (Phila Pa 1976) 2004;29:79-86.

9. Eyre DR, Benya P, Buckwalter J, et al. Intervertebral disk: basic science perspectives. In: Frymoyer JW, Gordon SL, editors. New perspectives on low back pain. Park Ridge: American Academy of Orthopaedic Surgeons; 1989. p.147-207.

10. White AA. Biomechanics of lumbar spine and sacroiliac articulation: relevance to idiopathic low back pain. In: White AA, Gordon SL, editors. Symposium on idiopathic low back pain. St. Louis: CV Mosby 
Co; 1981. p.296-322.

11. Buckwalter JA. Aging and degeneration of the human intervertebral disc. Spine (Phila Pa 1976) 1995;20: 1307-14.

12. World Health Organization. Obesity: preventing and managing the global epidemic. Report of a WHO consultation. Geneva: World Health Organization; 1997.

13. Vega GL. Results of expert meetings: obesity and cardiovascular disease. Obesity, the metabolic syndrome, and cardiovascular disease. Am Heart J 2001; 142:1108-16.

14. Field AE, Coakley EH, Must A, et al. Impact of overweight on the risk of developing common chronic diseases during a 10-year period. Arch Intern Med 2001;161:1581-6.

15. Fanuele JC, Abdu WA, Hanscom B, Weinstein JN. Association between obesity and functional status in patients with spine disease. Spine (Phila Pa 1976) 2002;27:306-12.

16. Dario AB, Ferreira ML, Refshauge KM, Lima TS, Ordonana JR, Ferreira PH. The relationship between obesity, low back pain, and lumbar disc degeneration when genetics and the environment are considered: a systematic review of twin studies. Spine J 2015;15: 1106-17.

17. Johnson WE, Stephan S, Roberts S. The influence of serum, glucose and oxygen on intervertebral disc cell growth in vitro: implications for degenerative disc disease. Arthritis Res Ther 2008;10:R46.

18. Sakellaridis N. The influence of diabetes mellitus on lumbar intervertebral disk herniation. Surg Neurol 2006;66:152-4.

19. Anekstein Y, Smorgick Y, Lotan R, et al. Diabetes mellitus as a risk factor for the development of lumbar spinal stenosis. Isr Med Assoc J 2010;12:16-20.

20. Centers for Disease Control and Prevention (CDC). State-specific prevalence of current cigarette smoking among adults and secondhand smoke rules and policies in homes and workplaces--United States, 2005. MMWR Morb Mortal Wkly Rep 2006;55:1148-51.

21. Sloan A, Hussain I, Maqsood M, Eremin O, ElSheemy M. The effects of smoking on fracture healing. Surgeon 2010;8:111-6.

22. Porter SE, Hanley EN Jr. The musculoskeletal effects of smoking. J Am Acad Orthop Surg 2001;9:9-17.

23. Castillo RC, Bosse MJ, MacKenzie EJ, Patterson BM.
Impact of smoking on fracture healing and risk of complications in limb-threatening open tibia fractures. J Orthop Trauma 2005;19:151-7.

24. Urban JP, Roberts S. Degeneration of the intervertebral disc. Arthritis Res Ther 2003;5:120-30.

25. Masuda K. Biological repair of the degenerated intervertebral disc by the injection of growth factors. Eur Spine J 2008;17 Suppl 4:441-51.

26. Zhao CQ, Wang LM, Jiang LS, Dai LY. The cell biology of intervertebral disc aging and degeneration. Ageing Res Rev 2007;6:247-61.

27. Freemont AJ. The cellular pathobiology of the degenerate intervertebral disc and discogenic back pain. Rheumatology (Oxford) 2009;48:5-10.

28. Adams MA, Roughley PJ. What is intervertebral disc degeneration, and what causes it? Spine (Phila Pa 1976) 2006;31:2151-61.

29. Battie MC, Videman T, Gill K, et al. 1991 Volvo Award in clinical sciences. Smoking and lumbar intervertebral disc degeneration: an MRI study of identical twins. Spine (Phila Pa 1976) 1991;16:1015-21.

30. An HS, Silveri CP, Simpson JM, et al. Comparison of smoking habits between patients with surgically confirmed herniated lumbar and cervical disc disease and controls. J Spinal Disord 1994;7:369-73.

31. Mattila VM, Saarni L, Parkkari J, Koivusilta L, Rimpela A. Early risk factors for lumbar discectomy: an 11-year follow-up of 57,408 adolescents. Eur Spine J 2008;17:1317-23.

32. Liuke M, Solovieva S, Lamminen A, et al. Disc degeneration of the lumbar spine in relation to overweight. Int J Obes (Lond) 2005;29:903-8.

33. Leboeuf-Yde C, Kjaer P, Bendix T, Manniche C. Selfreported hard physical work combined with heavy smoking or overweight may result in so-called Modic changes. BMC Musculoskelet Disord 2008;9:5.

34. Livshits G, Cohen Z, Higla O, Yakovenko K. Familial history, age and smoking are important risk factors for disc degeneration disease in Arabic pedigrees. Eur J Epidemiol 2001;17:643-51.

35. Liu YJ, Huang GS, Juan CJ, Yao MS, Ho WP, Chan WP. Intervertebral disk degeneration related to reduced vertebral marrow perfusion at dynamic contrast-enhanced MRI. AJR Am J Roentgenol 2009; 192:974-9.

36. Boubriak OA, Watson N, Sivan SS, Stubbens N, Urban JP. Factors regulating viable cell density in the 
intervertebral disc: blood supply in relation to disc height. J Anat 2013;222:341-8.

37. Holm S, Nachemson A. Nutrition of the intervertebral disc: acute effects of cigarette smoking. An experimental animal study. Ups J Med Sci 1988;93:919.

38. Iwahashi M, Matsuzaki H, Tokuhashi Y, Wakabayashi $\mathrm{K}$, Uematsu Y. Mechanism of intervertebral disc degeneration caused by nicotine in rabbits to explicate intervertebral disc disorders caused by smoking. Spine (Phila Pa 1976) 2002;27:1396-401.

39. Frymoyer JW, Pope MH, Clements JH, Wilder DG, MacPherson B, Ashikaga T. Risk factors in low-back pain: an epidemiological survey. J Bone Joint Surg Am 1983;65:213-8.

40. Jarvis MJ, Tunstall-Pedoe H, Feyerabend C, Vesey C, Saloojee Y. Comparison of tests used to distinguish smokers from nonsmokers. Am J Public Health 1987; 77:1435-8.

41. Rietbrock N, Kunkel S, Worner W, Eyer P. Oxygendissociation kinetics in the blood of smokers and non-smokers: interaction between oxygen and carbon monoxide at the hemoglobin molecule. Naunyn Schmiedebergs Arch Pharmacol 1992;345:123-8.

42. Akmal M, Kesani A, Anand B, Singh A, Wiseman M, Goodship A. Effect of nicotine on spinal disc cells: a cellular mechanism for disc degeneration. Spine (Phila Pa 1976) 2004;29:568-75.

43. Fogelholm RR, Alho AV. Smoking and intervertebral disc degeneration. Med Hypotheses 2001;56:537-9.

44. Vo N, Wang D, Sowa G, et al. Differential effects of nicotine and tobacco smoke condensate on human annulus fibrosus cell metabolism. J Orthop Res 2011;29:1585-91.

45. Oda $\mathrm{H}$, Matsuzaki $\mathrm{H}$, Tokuhashi $\mathrm{Y}$, Wakabayashi K, Uematsu Y, Iwahashi M. Degeneration of intervertebral discs due to smoking: experimental assessment in a rat-smoking model. J Orthop Sci 2004;9:135-41.

46. Nemoto Y, Matsuzaki H, Tokuhasi Y, et al. Histological changes in intervertebral discs after smoking and cessation: experimental study using a rat passive smoking model. J Orthop Sci 2006;11:191-7.

47. Uematsu Y, Matuzaki H, Iwahashi M. Effects of nicotine on the intervertebral disc: an experimental study in rabbits. J Orthop Sci 2001;6:177-82.

48. Gore DR, Carrera GF, Glaeser ST. Smoking and degenerative changes of the cervical spine: a roentgeno- graphic study. Spine J 2006;6:557-60.

49. Goldberg MS, Scott SC, Mayo NE. A review of the association between cigarette smoking and the development of nonspecific back pain and related outcomes. Spine (Phila Pa 1976) 2000;25:995-1014.

50. Flegal KM, Carroll MD, Ogden CL, Curtin LR. Prevalence and trends in obesity among US adults, 19992008. JAMA 2010;303:235-41.

51. Iatridis JC, MacLean JJ, Roughley PJ, Alini M. Effects of mechanical loading on intervertebral disc metabolism in vivo. J Bone Joint Surg Am 2006;88 Suppl 2:41-6.

52. Pietila TA, Stendel R, Kombos T, Ramsbacher J, Schulte T, Brock M. Lumbar disc herniation in patients up to 25 years of age. Neurol Med Chir (Tokyo) 2001;41:340-4.

53. Bostman OM. Prevalence of obesity among patients admitted for elective orthopaedic surgery. Int J Obes Relat Metab Disord 1994;18:709-13.

54. Mundt DJ, Kelsey JL, Golden AL, et al. An epidemiologic study of non-occupational lifting as a risk factor for herniated lumbar intervertebral disc. The Northeast Collaborative Group on Low Back Pain. Spine (Phila Pa 1976) 1993;18:595-602.

55. Videman T, Battie MC, Parent E, Gibbons LE, Vainio P, Kaprio J. Progression and determinants of quantitative magnetic resonance imaging measures of lumbar disc degeneration: a five-year follow-up of adult male monozygotic twins. Spine (Phila Pa 1976) 2008;33:1484-90.

56. Das UN. Is obesity an inflammatory condition? $\mathrm{Nu}$ trition 2001;17:953-66.

57. Ross R. Atherosclerosis: an inflammatory disease. N Engl J Med 1999;340:115-26.

58. Symmons DP, van Hemert AM, Vandenbroucke JP, Valkenburg HA. A longitudinal study of back pain and radiological changes in the lumbar spines of middle aged women. I. Clinical findings. Ann Rheum Dis 1991;50:158-61.

59. Samartzis D, Karppinen J, Chan D, Luk KD, Cheung $\mathrm{KM}$. The association of lumbar intervertebral disc degeneration on magnetic resonance imaging with body mass index in overweight and obese adults: a population-based study. Arthritis Rheum 2012;64: 1488-96.

60. Kaila-Kangas L, Leino-Arjas P, Riihimaki H, Luukkonen R, Kirjonen J. Smoking and overweight as 
predictors of hospitalization for back disorders. Spine (Phila Pa 1976) 2003;28:1860-8.

61. Takatalo J, Karppinen J, Taimela S, et al. Association of abdominal obesity with lumbar disc degeneration: a magnetic resonance imaging study. PLoS One 2013; 8:e56244.

62. Sivan SS, Tsitron E, Wachtel E, et al. Age-related accumulation of pentosidine in aggrecan and collagen from normal and degenerate human intervertebral discs. Biochem J 2006;399:29-35.

63. Metz LN, Lovell AF, Graham J, Liebenberg E, Havel P, Lotz JC. Does diabetes cause the intervertebral disc to degenerate? Spine J 2012;12 Suppl:S74-5.

64. Wagner DR, Reiser KM, Lotz JC. Glycation increases human annulus fibrosus stiffness in both experimental measurements and theoretical predictions. J Biomech 2006;39:1021-9.

65. Tang SY, Sharan AD, Vashishth D. Effects of collagen crosslinking on tissue fragility. Clin Biomech (Bristol, Avon) 2008;23:122-3.

66. Grunhagen T, Shirazi-Adl A, Fairbank JC, Urban JP. Intervertebral disk nutrition: a review of factors influencing concentrations of nutrients and metabolites. Orthop Clin North Am 2011;42:465-77.

67. Agius R, Galea R, Fava S. Bone mineral density and intervertebral disc height in type 2 diabetes. J Diabetes Complications 2016;30:644-50. 